Math. Nachr. 178 (1996), 81-101

\title{
Lipschitz - Orlicz Spaces and the Laplace Equation
}

By A.G. AksoY of Claremont and L.Maligranda of Luleå

Dedicated to Professor LARS ERIK PERsson on the Occasion of his $50^{\text {th }}$ birthday

(Received March 10, 1994)

Abstract. Stein and TAlbleson gave a characterization for $f \in L_{p}\left(\mathbb{R}^{n}\right)$ to be in the spaces $\operatorname{Lip}\left(\alpha, L_{p}\right)$ and $Z y g\left(\alpha, L_{p}\right)$ in terms of their Poisson integrals. In this paper we extend their results to Lipschitz-Orlicz spaces $\operatorname{Lip}\left(\varphi, L_{M}\right)$ and Zygmund-Orlicz spaces $Z y g\left(\varphi, L_{M}\right)$ and to the general function $\varphi \in P$ instead of the power function $\varphi(t)=t^{\alpha}$. Such results describe the behavior of the Laplace equation in terms of the smoothness property of differences of $f$ in Orlicz spaces $L_{M}\left(\mathbb{R}^{n}\right)$. More general spaces $\Lambda^{k}(\varphi, X, q)$ are also considered.

\section{Introduction}

The Poisson integral can be used to express the solution of the Dirichlet problem for the half-space $\mathbb{R}_{+}^{n+1}=\left\{(x, y): x \in \mathbb{R}^{n}, y>0\right\}:$ Let $f \in L_{p}\left(\mathbb{R}^{n}\right)$. Find a function $u(x, y)$ on $\mathbb{R}_{+}^{n+1}$ which is the solution of the Laplace equation

$$
\Delta u=\frac{\partial^{2} u}{\partial y^{2}}+\sum_{i=1}^{n} \frac{\partial^{2} u}{\partial x_{i}^{2}}=0 \quad\left(x \in \mathbb{R}^{n}, y>0\right)
$$

whose boundary values on $\mathbb{R}^{n}$ are $f(x)$. More precisely, if $f \in L_{p}\left(\mathbb{R}^{n}\right)$, then the Poisson integral of $f(x)$ is defined in $\mathbb{R}_{+}^{n+1}$ by

$$
\begin{aligned}
u(x, y) & =f(x) * P(x, y)=\int_{\mathbb{R}^{n}} f(x-z) P(z, y) d z \\
& =\int_{\mathbb{R}^{n}} f(z) P(x-z, y) d z
\end{aligned}
$$

1991 Mathematics Subject Classification. AMS subject classification (1991): Primary 46E30.

Keywords and phrases. Orlicz spaces, Lipschitz Condition, Zygmund Condition, Laplace Equation, convolution operator, Poisson integral, Banach function spaces.

This paper was done while the second named author visited the Claremont McKenna College in the period September-December 1993. 
The Poisson integral of $f(x)$ is the convolution of $f(x)$ with the Poisson kernel $P(x, y)$, which is defined by

$$
P(x, y)=\frac{1}{c_{n}} \cdot \frac{y}{\left(|x|^{2}+y^{2}\right)^{(n+1) / 2}} \quad\left(x \in \mathbb{R}^{n}, y>0\right),
$$

where $c_{n}=\pi^{(n+1) / 2} / \Gamma((n+1) / 2)$ is chosen so that $\int_{\mathbb{R}^{n}} P(x, y) d x=1$ for each $y>0$. STEIN [19] and TAIBLESON [20] gave a characterization for $f$ to be in the spaces $\operatorname{Lip}\left(\alpha, L_{p}\right)$ and $Z y g\left(\alpha, L_{p}\right)$ in terms of their Poissin integrals. Such results correlate the smoothness properties of functions from $L_{p}$ with the behavior of the solution of the Laplace equation. In their discussion they follow the earlier work of HARDY and LITTLEWOOD on the periodic spaces $\operatorname{Lip}\left(\alpha, L_{p}^{2 \pi}\right)$, and of ZYGMUND [24] on $2 \pi$ periodic smooth functions $Z y g\left(\alpha, L_{p}^{2 \pi}\right)$ (c.f. BUTzER-BERENs [3]). It should also be mentioned that TAIBLESON's paper [20] includes a discussion of the Laplace equation as well as the heat equation to be in $L_{p}\left(\mathbb{R}^{n}\right)$-spaces. There are many papers which investigate $\operatorname{Lip}\left(\alpha, L_{p}\right)$ in other directions, like Lorentz spaces $L_{p q}$ instead of $L_{p}$-spaces or $\operatorname{Lip}\left(\alpha, L_{p}\right)$ for negative $\alpha$ or $\Lambda(\alpha, p, q)$-spaces or $\Lambda^{k}(\varphi, X, q)$-spaces (see FLETT [5], Herz [6], Janson [7], Jones [8], Peetre [16], Stein [19], Taibleson [20] and TRIEBEL [21], [22]). These papers contain the problems of the duality, the equivalent norms and the interpolation spaces by the real and complex methods.

The purpose of this paper is to obtain the STEIN-TAIBLESON results for the LipschitzOrlicz spaces $\operatorname{Lip}\left(\varphi, L_{M}\right)$ and the Zygmund-Orlicz spaces $Z y g\left(\varphi, L_{M}\right)$, with a general functions $\varphi$ instead of the power function $\varphi(t)=t^{\alpha}$. A very rough description of the result would be that the derivative or the second derivative of a solution of the Laplace equation has a particular property if and only if $f$ has a very precise smoothness property describable in terms of differences of $f$ in the Orlicz spaces $L_{M}\left(\mathbb{R}^{n}\right)$.

The Orlicz space

$$
\begin{aligned}
L_{M}=L_{M}\left(\mathbb{R}^{n}\right) & =\left\{f \in L_{0}\left(\mathbb{R}^{n}\right)\right. \text { such that } \\
I_{M}(\lambda f) & \left.:=\int_{\mathbb{R}^{n}} M(\lambda|f(x)|) d x<\infty \text { for some } \lambda>0\right\}
\end{aligned}
$$

is a Banach space with the Luxemburg-Nakano norm

$$
\|f\|_{M}=\inf \left\{\lambda>0: I_{M}(f / \lambda) \leq 1\right\},
$$

where $L^{0}\left(\mathbb{R}^{n}\right)$ denotes the space of all (equivalence classes of ) Lebesgue measurable real functions on $\mathbb{R}^{n}$ and $M:[0, \infty) \rightarrow[0, \infty)$ is a Young function, i.e., a convex nondecreasing function vanishing at zero (not identically 0 or $\infty$ on $(0, \infty)$ ) (see [9], $[13],[17])$.

Let $P$ be the class of functions $\varphi:[0, \infty) \rightarrow[0, \infty)$ which are continuous nondecreasing and zero only at 0 . For $\varphi \in P$, let us consider the Lipschitz-Orlicz space:

$$
\begin{aligned}
\operatorname{Lip}\left(\varphi, L_{M}\right)= & \left\{f \in L_{M}\left(\mathbb{R}^{n}\right)\right. \text { such that } \\
& \left.\|f(x+h)-f(x)\|_{M} \leq C \varphi(|h|) \text { for all }|h|>0\right\},
\end{aligned}
$$

and the Zygmund-Orlicz space

$$
\begin{aligned}
Z y g\left(\varphi, L_{M}\right)= & \left\{f \in L_{M}\left(\mathbb{R}^{n}\right)\right. \text { such that } \\
& \left.\|f(x+h)+f(x-h)-2 f(x)\|_{M} \leq D \varphi(|h|) \text { for all }|h|>0\right\} .
\end{aligned}
$$


Both spaces $\operatorname{Lip}\left(\varphi, L_{M}\right)$ and $Z y g\left(\varphi, L_{M}\right)$ are Banach spaces with the norms

$$
\|f\|_{M}+\sup _{|h|>0} \frac{\|f(x+h)-f(x)\|_{M}}{\varphi(|h|)}
$$

and

$$
\|f\|_{M}+\sup _{|h|>0} \frac{\|f(x+h)+f(x-h)-2 f(x)\|_{M}}{\varphi(|h|)},
$$

respectively. Clearly $\operatorname{Lip}\left(\varphi, L_{M}\right) \subset Z y g\left(\varphi, L_{M}\right)$.

We will need to put restrictions on the growth of the function $\varphi \in P$. For $\varphi \in P$ we can define the so called indices of $\varphi$ (cf. [10], [12], [13]):

$$
\alpha_{\varphi}=\lim _{t \rightarrow 0} \frac{\ln s_{\varphi}(t)}{\ln t}, \quad \beta_{\varphi}=\lim _{t \rightarrow \infty} \frac{\ln s_{\varphi}(t)}{\ln t}
$$

where

$$
s_{\varphi}(t)=\sup _{s>0} \frac{\varphi(s t)}{\varphi(s)}
$$

Obviously $0 \leq \alpha_{\varphi} \leq \beta_{\varphi}$ for $\varphi \in P$. For the power function $\varphi(t)=t^{a}$ we have $\alpha_{\varphi}=\beta_{\varphi}=a$.

This paper is organized as follows. In Section 2 we characterize the functions from the Lipschitz class $\operatorname{Lip}\left(\varphi, L_{M}\right)$ in terms of the derivatives of their Poisson integrals. In Section 3 a similar characterization is given for the Zygmund class $Z y g\left(\varphi, L_{M}\right)$. In Section 4 we consider the more general spaces $\Lambda^{k}(\varphi, X, q), k=1,2$, and prove some results about them. For example, for $0<\alpha_{\varphi} \leq \beta_{\varphi}<k,(k=1,2), f \in \Lambda^{k}(\varphi, X, q)$ if and only if the solution $u$ of the Laplace equation satisfies

$$
\frac{y^{k}}{\varphi(y)}\left\|\frac{\partial^{k} u(x, y)}{\partial y^{k}}\right\|_{X} \in L_{q}\left((0, \infty), \frac{d y}{y}\right)
$$

This section also contain some additional remarks.

\section{The Lipschitz Condition}

In the proof of the main theorem of this section we will need the following equivalence property between indices and integrals of $\varphi \in P$ (the proof of these equivalences can be found in [10], [12] or in [13], Th. 11.8):

Let $\varphi \in P, s_{\varphi}(t)<\infty$ for every $t>0$, and $r>0$. Then

$$
\alpha_{\varphi}>0 \text { if and only if } \int_{0}^{t} \frac{\varphi(s)}{s} d s \leq A \varphi(t) \text { for all } t>0
$$

and

$$
\beta_{\varphi}<r \text { if and only if } \int_{0}^{\infty} \frac{\varphi(s)}{s^{r+1}} d s \leq B \frac{\varphi(t)}{t^{r}} \text { for all } t>0
$$


Theorem 2.1. Let $f \in L_{M}\left(\mathbb{R}^{n}\right)$ and $u(x, y)=\int_{\mathbb{R}^{n}} f(x-z) P(z, y) d z$ be its Poisson integral. If $0<\alpha_{\varphi} \leq \beta_{\varphi}<1$, then the following are equivalent:

(i) $f \in \operatorname{Lip}\left(\varphi, L_{M}\right)$,

(ii) $\left\|\frac{\partial}{\partial y} u(x, y)\right\|_{M} \leq C \varphi(y) / y$ for all $y>0$,

(iii) $\|u(x, y)-f(x)\|_{M} \leq D \varphi(y)$ for all $y>0$.

Proof . (i) $\Longrightarrow$ (ii). Let $f \in \operatorname{Lip}\left(\varphi, L_{M}\right)$. Since

$$
\begin{aligned}
\frac{\partial P(x, y)}{\partial y} & =\frac{1}{c_{n}} \cdot \frac{|x|^{2}-n y^{2}}{\left(|x|^{2}+y^{2}\right)^{(n+3) / 2}} \\
& =\frac{1}{c_{n}}\left[\frac{1}{\left(|x|^{2}+y^{2}\right)^{(n+1) / 2}}-\frac{(n+1) y^{2}}{\left(|x|^{2}+y^{2}\right)^{(n+3) / 2}}\right],
\end{aligned}
$$

it follows that

$$
\begin{gathered}
\int_{\mathbb{R}^{n}} \frac{\partial P(x, y)}{\partial y} d x=0 \\
\left|\frac{\partial P(x, y)}{\partial y}\right| \leq \frac{n}{c_{n}} \min \left\{|x|^{-n-1}, y^{-n-1}\right\}
\end{gathered}
$$

and

$$
\left|\frac{\partial P(x, y)}{\partial y}\right| \leq n \frac{P(x, y)}{y} .
$$

Then, since the integral defining the convolution converges absolutely, we can write

$$
\begin{aligned}
\frac{\partial}{\partial y} u(x, y) & =\int_{\mathbb{R}^{n}} \frac{\partial}{\partial y} P(z, y) f(x-z) d z \\
& =\int_{\mathbb{R}^{n}} \frac{\partial}{\partial y} P(z, y)[f(x-z)-f(x)] d z
\end{aligned}
$$

and by the generalized Minkowski inequality (cf. [10], pp. 45-46)

$$
\begin{aligned}
\left\|\frac{\partial}{\partial y} u(x, y)\right\|_{M} \leq & \int_{\mathbb{R}^{n}}\left|\frac{\partial}{\partial y} P(z, y)\right|\|f(x-z)-f(x)\|_{M} d z \\
\leq & \frac{n}{c_{n}} y^{-n-1} \int_{|z| \leq y}\|f(x-z)-f(x)\|_{M} d z \\
& +\frac{n}{c_{n}} \cdot \int_{|z|>y}|z|^{-n-1}\|f(x-z)-f(x)\|_{M} d z .
\end{aligned}
$$

Next, set $z=r \xi \in \mathbb{R}^{n}$ with $r=|z|$ and $|\xi|=1$. Then, with

$$
\|f(x-z)-f(x)\|_{M}=\omega_{M}(z)=\omega_{M}(r \xi)
$$

and

$$
\Omega(r)=\int_{S^{n-1}} \omega_{M}(r \xi) d \sigma(\xi)
$$


the inequality above becomes (because $d z=d \xi r^{n-1} d r$ )

$$
\left\|\frac{\partial}{\partial y} u(x, y)\right\|_{M} \leq \frac{n}{c_{n}}\left[y^{-n-1} \int_{0}^{y} \Omega(r) r^{n-1} d r+\int_{y}^{\infty} \Omega(r) r^{-2} d r\right]
$$

The assumption that $f \in \operatorname{Lip}\left(\varphi, L_{M}\right)$ gives

$$
\Omega(r) \leq C \varphi(r) \sigma\left(S^{n-1}\right)=2 c_{n-1} C \varphi(r)
$$

and by the assumption $\beta_{\varphi}<1$, in the equivalent form (2.2), we obtain

$$
\begin{aligned}
\left\|\frac{\partial}{\partial y} u(x, y)\right\|_{M} & \leq \frac{2 n C c_{n-1}}{c_{n}}\left[y^{-n-1} \int_{0}^{y} \varphi(r) r^{n-1} d r+\int_{y}^{\infty} \varphi(r) r^{-2} d r\right] \\
& \leq \frac{2 n C c_{n-1}}{c_{n}}\left[\frac{1}{n} \cdot \frac{\varphi(y)}{y}+B \frac{\varphi(y)}{y}\right] \\
& \leq C_{1} \frac{\varphi(y)}{y}
\end{aligned}
$$

Proof. (ii) $\Longrightarrow$ (iii). For a.e. $x \in \mathbb{R}^{n}$, it yields that

$$
u(x, y)-f(x)=\int_{0}^{y} \frac{\partial u(x, s)}{\partial s} d s
$$

By the generalized Minkowski inequality and the assumption $\alpha_{\varphi}>0$, in the equivalent form (2.1), we obtain

$$
\begin{aligned}
\|u(x, y)-f(x)\|_{M} & \leq \int_{0}^{y}\left\|\frac{\partial u(x, s)}{\partial s}\right\|_{M} d s \\
& \leq C \int_{0}^{y} \frac{\varphi(s)}{s} d s \\
& \leq C A \varphi(y) .
\end{aligned}
$$

Proof . (iii) $\Longrightarrow$ (ii). First, note that

$$
\left\|\frac{\partial}{\partial y} u(x, y)\right\|_{M}=\lim _{m \rightarrow \infty}\left\|\frac{\partial}{\partial y}\left[u(x, y)-u\left(x, 2^{m} y\right)\right]\right\|_{M}
$$

Thus, using the fact that the convolution operator is bounded from $L_{M}\left(\mathbb{R}^{n}\right) \times L_{1}\left(\mathbb{R}^{n}\right)$ into $L_{M}\left(\mathbb{R}^{n}\right)$ with norm less or equal than 1 (cf. Lemma 4.1 ), the above property of 
the Poisson kernel and the assumption $\beta_{\varphi}<1$, in the equivalent form (2.2), we obtain

$$
\begin{aligned}
& \left\|\frac{\partial}{\partial y}\left[u(x, y)-u\left(x, 2^{m} y\right)\right]\right\|_{M} \\
\leq & \sum_{k=1}^{m}\left\|\frac{\partial}{\partial y}\left[u\left(x, 2^{k-1} y\right)-u\left(x, 2^{k} y\right)\right]\right\|_{M} \\
= & \sum_{k=1}^{m}\left\|\left[f(x)-u\left(x, 2^{k-1} y\right)\right] * \frac{\partial}{\partial y}\left(P, 2^{k-1} y\right)\right\|_{M} \\
\leq & \sum_{k=1}^{m}\left\|f(x)-u\left(x, 2^{k-1} y\right)\right\|_{M}\left\|\frac{\partial}{\partial y} P\left(x, 2^{k-1} y\right)\right\|_{1} \\
\leq & n \sum_{k=1}^{m}\left\{\left\|f(x)-u\left(x, 2^{k-1} y\right)\right\|\left\|_{M}\right\| P\left(x, 2^{k-1} y\right) \|_{1} /\left(2^{k-1} y\right)\right\} \\
\leq & n \sum_{k=1}^{m}\left\|f(x)-u\left(x, 2^{k-1} y\right)\right\| \|_{M} /\left(2^{k-1} y\right) \\
\leq & n D \sum_{k=1}^{m} \varphi\left(2^{k-1} y\right) /\left(2^{k-1} y\right) \leq 2 n D \sum_{k=1}^{m} \int_{2^{k-1} y}^{2^{k} y} \varphi(s) s^{-2} d s \\
\leq & 2 n D \int_{y}^{\infty} \varphi(s) s^{-2} d s \leq 2 n D B \varphi(y) / y .
\end{aligned}
$$

Proof . (ii) $\Longrightarrow$ (i). First, we prove the following lemma.

Lemma 2.2. Let $f \in L_{M}\left(\mathbb{R}^{n}\right)$ and $u(x, y)=\int_{\mathbb{R}^{n}} f(x-z) P(z, y) d z$ be its Poisson integral. If $0<\alpha_{\varphi} \leq \beta_{\varphi}<1$, then for all $y>0$

$$
\left\|\frac{\partial}{\partial y} u(x, y)\right\|_{M} \leq C \varphi(y) / y
$$

if and only if for all $y>0$ and for each $i=1,2, \ldots, n$

$$
\left\|\frac{\partial}{\partial x_{i}} u(x, y)\right\|_{M} \leq C^{\prime} \varphi(y) / y .
$$

The smallest $C$ in (2.3) is comparable to the smallest $C^{\prime}$ in (2.4).

P r o of of Lemma 2.2. First we prove that if $y_{1}, y_{2}>0$, then

$$
u\left(x, y_{1}+y_{2}\right)=u\left(x, y_{2}\right) * P\left(x, y_{1}\right)
$$

and

$$
\frac{\partial^{2} u}{\partial y \partial x_{i}}\left(x, y_{1}+y_{2}\right)=\frac{\partial u}{\partial y}\left(x, y_{2}\right) * \frac{\partial P}{\partial x_{i}}\left(x, y_{1}\right), \quad i=1,2, \ldots, n
$$

Since

$$
\int_{\mathbb{R}^{n}} P\left(x, y_{1}+y_{2}\right)^{p} d x \leq C_{2} y_{1}^{-(p-1) n} \quad(p>1)
$$


for all $y_{2}>0$, it follows that the Poisson kernel $P\left(x, y_{1}+y_{2}\right)$ has the Poisson integral in $\mathbb{R}^{n} \times\left(y_{1}, \infty\right)$

$$
P\left(x, y_{1}+y_{2}\right)=\int_{\mathbb{R}^{n}} P\left(s, y_{1}\right) P\left(s-z, y_{2}\right) d s=P\left(x, y_{1}\right) * P\left(x, y_{2}\right)
$$

Then

$$
\begin{aligned}
u\left(x, y_{1}+y_{2}\right) & =f(x) * P\left(x, y_{1}+y_{2}\right)=\int_{\mathbb{R}^{n}} f(x) P\left(x-z, y_{1}+y_{2}\right) d z \\
& =\int_{\mathbb{R}^{n}} f(z) \int_{\mathbb{R}^{n}} P\left(s, y_{1}\right) P\left(x-z-s, y_{2}\right) d s d z \\
& =\int_{\mathbb{R}^{n}} f(z) \int_{\mathbb{R}^{n}} P\left(x-t, y_{1}\right) P\left(t-z, y_{2}\right) d t d z \\
& =\int_{\mathbb{R}^{n}}\left\{\int_{\mathbb{R}^{n}} f(z) P\left(t-z, y_{2}\right) d z\right\} P\left(x-t, y_{1}\right) d t \\
& =\int_{\mathbb{R}^{n}} u\left(t, y_{2}\right) P\left(x-t, y_{1}\right) d t
\end{aligned}
$$

that is, $u\left(x, y_{1}+y_{2}\right)=u\left(x, y_{2}\right) * P\left(x, y_{1}\right)$, and the equality (2.5) is proved.

For fixed $y_{1}>0$, we have, according to the equality (2.5),

$$
u\left(x, y_{1}+y\right)=u(x, y) * P\left(x, y_{1}\right)
$$

Differentiating we obtain

$$
\frac{\partial}{\partial y} u\left(x, y+y_{1}\right)=\frac{\partial}{\partial y} u(x, y) * P\left(x, y_{1}\right)
$$

which can be expressed as

$$
\frac{\partial}{\partial y} u\left(x, y_{1}+y_{2}\right)=\frac{\partial u}{\partial y}\left(x, y_{1}\right) * P\left(x, y_{2}\right)=\int_{\mathbb{R}^{n}} \frac{\partial}{\partial y} u\left(z, y_{1}\right) P\left(x-z, y_{2}\right) d z
$$

Therefore

$$
\begin{aligned}
\frac{\partial^{2} u}{\partial y \partial x_{i}}\left(x, y_{1}+y_{2}\right) & =\int_{\mathbb{R}^{n}} \frac{\partial u}{\partial y}\left(z, y_{1}\right) \frac{\partial P}{\partial x_{i}}\left(x-z, y_{2}\right) d z \\
& =\frac{\partial u}{\partial y}\left(x, y_{2}\right) * \frac{\partial P}{\partial x_{i}}\left(x, y_{1}\right)
\end{aligned}
$$

and also the equality (2.6) is proved. Taking $y_{1}=y_{2}=y / 2$ in the equality (2.6), we obtain

$$
\frac{\partial^{2} u}{\partial y \partial x_{i}}(x, y)=\frac{\partial u}{\partial y}(x, y / 2) * \frac{\partial P}{\partial x_{i}}(x, y / 2)
$$

and so (cf. Lemma 4.1)

$$
\left\|\frac{\partial^{2} u}{\partial y \partial x_{i}}(x, y)\right\|_{M} \leq\left\|\frac{\partial u}{\partial y}(x, y / 2)\right\|_{M}\left\|\frac{\partial P}{\partial x_{i}}(x, y / 2)\right\|_{1} .
$$


Now, if (2.3) holds, then

$$
\left\|\frac{\partial u}{\partial y}(x, y / 2)\right\|_{M} \leq 2 C \varphi(y / 2) / y \leq 2 C \varphi(y) / y .
$$

For the Poisson kernel we have

$$
\frac{\partial P(x, y)}{\partial x_{i}}=\frac{1}{c_{n}} \cdot \frac{-(n+1) x_{i} y}{\left(|x|^{2}+y^{2}\right)^{(n+3) / 2}}, \quad i=1,2, \ldots, n,
$$

and so

$$
\left|\frac{\partial P(x, y)}{\partial x_{i}}\right| \leq \frac{n+1}{2} \cdot \frac{P(x, y)}{y} \quad i=1,2, \ldots, n,
$$

which means that

$$
\left\|\frac{\partial P}{\partial x_{i}}(x, y / 2)\right\|_{1} \leq(n+1)\left\|\frac{P(x, y / 2)}{y}\right\|_{1} \leq(n+1) \frac{1}{y} .
$$

Substituting these estimates into (2.5) we obtain

$$
\left\|\frac{\partial^{2}}{\partial y \partial x_{i}} u(x, y)\right\|_{M} \leq 2 C(n+1) \varphi(y) / y^{2} .
$$

On the other hand, using the fact that the convolution operator is bounded from $L_{M}\left(\mathbb{R}^{n}\right) \times L_{1}\left(\mathbb{R}^{n}\right)$ into $L_{M}\left(\mathbb{R}^{n}\right)$ with the norm less or equal to 1 (cf. Lemma 4.1 ) and the above property of the Poisson kernel, we obtain

$$
\begin{aligned}
\left\|\frac{\partial}{\partial x_{i}} u(x, y)\right\|_{M} & =\left\|f(x) * \frac{\partial P(x, y)}{\partial x_{i}}\right\|_{M} \leq\|f(x)\|_{M}\left\|\frac{\partial P(x, y)}{\partial x_{i}}\right\|_{1} \\
& \leq \frac{n+1}{2} \cdot \frac{1}{y}\|P(x, y)\|_{1}\|f(x)\|_{M}=\frac{n+1}{2} \cdot \frac{1}{y}\|f(x)\|_{M},
\end{aligned}
$$

which implies that $\frac{\partial}{\partial x_{i}} u(x, y) \rightarrow 0$ as $y \rightarrow \infty$. Therefore

$$
\frac{\partial}{\partial x_{i}} u(x, y)=-\int_{y}^{\infty} \frac{\partial^{2} u}{\partial y \partial x_{i}}(x, s) d s
$$

and, by (2.8), (2.2),

$$
\begin{aligned}
\left\|\frac{\partial}{\partial x_{i}} u(x, y)\right\|_{M} & \leq \int_{y}^{\infty}\left\|\frac{\partial^{2} u}{\partial y \partial x_{i}}(x, s)\right\|_{M} d s \\
& \leq 2 C(n+1) \int_{y}^{\infty} s^{-2} \varphi(s) d s \\
& \leq 2 B C(n+1) \varphi(y) / y .
\end{aligned}
$$

Conversely, if (2.5) holds, then in the same way as before we obtain

$$
\left\|\frac{\partial^{2} u}{\partial x_{i}^{2}}\right\|_{M} \leq C_{3} \varphi(y) / y^{2}, \quad i=1,2, \ldots, n .
$$


Since $u$ is harmonic, that is,

$$
\frac{\partial^{2}}{\partial y^{2}} u(x, y)=-\sum_{i=1}^{n} \frac{\partial^{2}}{\partial x_{i}^{2}} u(x, y)
$$

we therefore have

$$
\left\|\frac{\partial^{2}}{\partial y^{2}} u(x, y)\right\|_{M} \leq C_{3} \varphi(y) / y^{2}
$$

and a similar integration argument then shows that

$$
\left\|\frac{\partial u}{\partial y}\right\|_{M} \leq C_{4} \varphi(y) / y
$$

Proof. (ii) $\Longrightarrow$ (i). Assume that $f \in L_{M}$ and that the condition (2.3) holds. For $h \in \mathbb{R}^{n}$ and $0<y<|h|$ we have

$$
\begin{aligned}
& u(x+h, y)-u(x, y) \\
= & \int_{y}^{|h|} \frac{\partial u(x, s)}{\partial s} d s+\int_{0}^{h_{1}} \frac{\partial u\left(x_{1}+z_{1}, x_{2}, x_{3}, \ldots, x_{n},|h|\right)}{\partial z_{1}} d z_{1} \\
& +\int_{0}^{h_{2}} \frac{\partial u\left(x_{1}+h_{1}, x_{2}+z_{2}, x_{3}, \ldots, x_{n},|h|\right)}{\partial z_{2}} d z_{2}+\ldots \\
& +\int_{0}^{h_{n}} \frac{\partial u\left(x_{1}+h_{1}, x_{2}+h_{2}, x_{3}+h_{3}, \ldots, x_{n}+z_{n},|h|\right)}{\partial z_{n}} d z_{n} \\
& +\int_{|h|}^{y} \frac{\partial u(x+h, s)}{\partial s} d s,
\end{aligned}
$$

and so

$$
\begin{aligned}
\|u(x+h, y)-u(x, y)\|_{M} \leq & 2 \int_{y}^{|h|}\left\|\frac{\partial u(x, s)}{\partial s}\right\|_{M} d s \\
& +\sum_{i=1}^{n} \int_{0}^{\left|h_{i}\right|}\left\|\frac{\partial u}{\partial x_{i}}(x,|h|)\right\|_{M} d z_{i}
\end{aligned}
$$

Using the assumption (2.3), Lemma 2.2 and property (2.1), the last expression becomes less or equal to

$$
2 C \int_{y}^{|h|} \frac{\varphi(s)}{s} d s+C^{\prime} \sum_{i=1}^{n} \int_{0}^{\left|h_{i}\right|} \frac{\varphi(|h|)}{|h|} d z_{i} \leq 2 A C \varphi(|h|)+C^{\prime} \frac{\varphi(|h|)}{|h|} \sum_{i=1}^{n}\left|h_{i}\right|
$$

which is less or equal to

$$
C_{5} \varphi(|h|)
$$

Now, since $u(x, y) \rightarrow f(x)$ for almost all $x \in \mathbb{R}^{n}$ when $y \rightarrow 0^{+}$, we obtain (by the Fatou Lemma) that $f \in \operatorname{Lip}\left(\varphi, L_{M}\right)$. This completes the proof of the theorem. 
Corollary 2.3. If $0<\alpha_{\varphi} \leq \beta_{\varphi} \leq 1$, then $\operatorname{Lip}\left(\varphi, L_{M}\right)=Z y g\left(\varphi, L_{M}\right)$.

Proof It is enough to prove the imbedding $Z y g\left(\varphi, L_{M}\right) \subset \operatorname{Lip}\left(\varphi, L_{M}\right)$. Let $f \in Z y g\left(\varphi, L_{M}\right)$. Then for $u(x, y)=f(x) * P(x, y)$ we have

$$
\begin{aligned}
\frac{\partial}{\partial y} u(x, y) & =\int_{\mathbb{R}^{n}} \frac{\partial P}{\partial y}(z, y) f(x-z) d z \\
& =\frac{1}{2} \int_{\mathbb{R}^{n}} \frac{\partial P}{\partial y}(z, y)[f(x+z)+f(x-z)-2 f(x)] d z,
\end{aligned}
$$

and, by the generalized Minkowiski inequality (cf. [10] ),

$$
\begin{aligned}
\left\|\frac{\partial}{\partial y} u(x, y)\right\|_{M} & \leq \frac{1}{2} \int_{\mathbb{R}^{n}}\left|\frac{\partial P}{\partial y}(z, y)\right|\|f(x+z)+f(x-z)-2 f(x)\|_{M} d z \\
& \leq \frac{1}{2} D \int_{\mathbb{R}^{n}}\left|\frac{\partial P}{\partial y}(z, y)\right| \varphi(|z|) d z,
\end{aligned}
$$

and by using the estimates from the proof of Theorem 2.1, we obtain

$$
\left\|\frac{\partial}{\partial y} u(x, y)\right\|_{M} \leq C_{6} y^{-1} \varphi(y) \text { for all } y>0,
$$

which, according to Theorem 2.1, gives $f \in \operatorname{Lip}\left(\varphi, L_{M}\right)$.

Remark 2.4. Theorem 2.1 in the case of $L_{p}\left(\mathbb{R}^{n}\right)$-space $(1 \leq p \leq \infty)$ and with $\varphi(t)=t^{\alpha}$, where $0<\alpha<1$, was proved by STEIN ([19], Prop. 7, $\left.7^{\prime}\right)$ and by TAIBLESON ([20], Th. 4).

Remark 2.5. Using the fact that

$$
|P(x, y)| \leq \frac{1}{c_{n}}\left\{\min \left\{y^{-n}, y|x|^{-n-1}\right\}\right\},
$$

we can prove for $f \in \operatorname{Lip}\left(\varphi, L_{M}\right)$, in a similar way as in the proof of Theorem 2.1, that

$$
\begin{aligned}
\|u(x, y)-f(x)\|_{M} \leq & \int_{\mathbb{R}^{n}}\|f(x-z)-f(x)\|_{M}|P(z, y)| d z \\
\leq & \frac{1}{c_{n}} y^{-n} \int_{|z| \leq y}\|f(x-z)-f(x)\|_{M} d z \\
& +\frac{1}{c_{n}} \int_{|z| \geq y} y|z|^{-n-1}\|f(x-z)-f(x)\|_{M} d z \\
\leq & \frac{2 n C c_{n-1}}{c_{n}}\left[y^{-n} \int_{0}^{y} \varphi(r) r^{n-1} d r+y \int_{y}^{\infty} \varphi(r) r^{-2} d r\right] .
\end{aligned}
$$

\section{The Zygmund Condition}

The next result is the case of Zygmund condition in Orlicz spaces which gives the Zygmund- Orlicz spaces $Z y g\left(\varphi, L_{M}\right)$. ZYGMUND [23] introduced spaces of smooth functions $Z y g\left(1, L_{p}\right)$. 
Theorem 3.1. Let $f \in L_{M}\left(\mathbb{R}^{n}\right)$ und $u(x, y)=\int_{\mathbb{R}^{n}} f(x-z) P(z, y) d z$ be its Poisson integral. If $0<\alpha_{\varphi} \leq \beta_{\varphi}<2$, then $f \in Z y g\left(\varphi, L_{M}\right)$ if and only if

$$
\left\|\frac{\partial^{2}}{\partial y^{2}} u(x, y)\right\|_{M} \leq C \varphi(y) / y^{2} \text { for all } y>0 \text {. }
$$

Proof (Necessity). Assume that $f \in Z y g\left(\varphi, L_{M}\right)$. Since

$$
\begin{aligned}
\frac{\partial^{2} P(x, y)}{\partial y^{2}} & =\frac{n+1}{c_{n}} \cdot \frac{y\left(n y^{2}-3|x|^{2}\right)}{\left(|x|^{2}+y^{2}\right)^{(n+5) / 2}} \\
& =\frac{n+1}{c_{n}}\left[\frac{(n+3) y^{3}}{\left(|x|^{2}+y^{2}\right)^{(n+5) / 2}}-\frac{3 y}{\left(|x|^{2}+y^{2}\right)^{(n+3) / 2}}\right]
\end{aligned}
$$

it follows that

$$
\begin{gathered}
\int_{\mathbb{R}^{x}} \frac{\partial^{2} P(x, y)}{\partial y^{2}} d x=0 \\
\left|\frac{\partial^{2} P(x, y)}{\partial y^{2}}\right| \leq \frac{(n+1)(n+2)}{c_{n}} \cdot\left\{\min \left\{|x|^{-n-2} y, y^{-n-2}\right\}\right\}
\end{gathered}
$$

and

$$
\begin{aligned}
\left|\frac{\partial^{2} P(x, y)}{\partial y^{2}}\right| & \leq \frac{(n+1)(n+2)}{c_{n}} \cdot \frac{y}{\left(|x|^{2}+y^{2}\right)^{(n+3) / 2}} \\
& \leq(n+1)(n+2) y^{-2} P(x, y) .
\end{aligned}
$$

Then we can write

$$
\begin{aligned}
\frac{\partial^{2}}{\partial y^{2}} u(x, y) & =\int_{\mathbb{R}^{n}} \frac{\partial^{2} P}{\partial y^{2}}(z, y) f(x-z) d z \\
& =\frac{1}{2} \int_{\mathbb{R}^{n}} \frac{\partial^{2} P}{\partial y^{2}}(z, y)[f(x+z)+f(x-z)-2 f(x)] d z
\end{aligned}
$$

Using the generalized Minkovski inequality we obtain

$$
\begin{aligned}
\left\|\frac{\partial^{2}}{\partial y^{2}} u(x, y)\right\|_{M} \leq & \frac{1}{2} \int_{\mathbb{R}^{n}}\left|\frac{\partial^{2} P}{\partial y^{2}}(z, y)\right|\|f(x+z)+f(x-z)-2 f(x)\|_{M} d z \\
\leq & \frac{1}{2} \cdot \frac{(n+1)(n+2)}{c_{n}} y^{-n-2} \\
& \times \int_{|z| \leq y}\|f(x+z)+f(x-z)-2 f(x)\|_{M} d z \\
& +\frac{1}{2} \cdot \frac{(n+1)(n+2)}{c_{n}} y \\
& \times \int_{|z|>y}|z|^{-n-3}\|f(x+z)+f(x-z)-2 f(x)\|_{M} d z .
\end{aligned}
$$

Next set $z=r \xi \in \mathbb{R}^{n}$, with $r=|z|$ and $|\xi|=1$. Then, with

$$
\|f(x+z)+f(x-z)-2 f(x)\|_{M}=\omega_{M}(z)=\omega_{M}(r \xi)
$$


and

$$
\Omega(r)=\int_{S^{n-1}} \omega_{M}(r \xi) d \sigma(\xi),
$$

the inequality above becomes (because $d z=d \xi r^{n-1} d r$ )

$$
\begin{aligned}
\left\|\frac{\partial^{2}}{\partial y^{2}} u(x, y)\right\|_{M} \leq & \frac{1}{2} \cdot \frac{(n+1)(n+2)}{c_{n}}\left[y^{-n-2} \int_{0}^{y} \Omega(r) r^{n-1} d r\right. \\
& \left.+y \int_{y}^{\infty} \Omega(r) r^{-4} d r\right] .
\end{aligned}
$$

The assumption $f \in Z y g\left(\varphi, L_{M}\right)$ gives

$$
\Omega(r) \leq D \varphi(r) \sigma\left(S^{n-1}\right)=2 c_{n-1} D \varphi(r)
$$

and, by the assumption $\beta_{\varphi}<2$, in the equivalent form (2.2), we obtain

$$
\begin{aligned}
\left\|\frac{\partial^{2}}{\partial y^{2}} u(x, y)\right\|_{M} & \leq C_{7}\left[y^{-n-2} \int_{0}^{y} \Omega(r) r^{n-1} d r+y \int_{y}^{\infty} \varphi(r) r^{-4} d r\right] \\
& \leq C_{7}\left[y^{-n-2} \int_{0}^{y} \Omega(r) r^{n-1} d r+\int_{y}^{\infty} \varphi(r) r^{-3} d r\right] \\
& \leq C_{8} y^{-2} \varphi(y) .
\end{aligned}
$$

P r o of (Suficiency). First we prove the following lemma.

Lemma 3.2. Let $f \in L_{M}\left(\mathbb{R}^{n}\right)$ und $u(x, y)=\int_{\mathbb{R}^{n}} f(x-z) P(z, y) d z$ be its Poisson integral. If $0<\alpha_{\varphi} \leq \beta_{\varphi}<2$, then the following conditions are equivalent:

(a) $\left\|\frac{\partial^{2}}{\partial y^{2}} u(x, y)\right\|_{M} \leq C \varphi(y) / y^{2}$ for all $y>0$,

(b) $\left\|\frac{\partial^{2} u}{\partial y \partial x_{i}}(x, y)\right\|_{M} \leq D y^{-2} \varphi(y)$ for all $y>0$ and each $i=1,2, \ldots, n$,

(c) $\left\|\frac{\partial^{2} u}{\partial x_{i} \partial x_{j}}(x, y)\right\|_{M} \leq E y^{-2} \varphi(y)$ for all $y>0$ and each $i, j=1,2, \ldots, n$.

Proof . (a) $\Rightarrow$ (b). Differentiating equality (2.5) we obtain

$$
\frac{\partial^{3} u}{\partial y^{2} \partial x_{i}}\left(x, y+y_{1}\right)=\frac{\partial^{2} u}{\partial y^{2}}(x, y) * \frac{\partial P}{\partial x_{i}}\left(x, y_{1}\right)
$$

Then, by arguing in a similar way as in the proof of Lemma 2.2, we find that

$$
\begin{aligned}
\left\|\frac{\partial^{3} u}{\partial y^{2} \partial x_{i}}(x, y)\right\|_{M} & =\left\|\frac{\partial^{2} u}{\partial y^{2}}(x, y / 2) * \frac{\partial P}{\partial x_{i}}(x, y / 2)\right\|_{M} \\
& \leq\left\|\frac{\partial^{2} u}{\partial y^{2}}(x, y / 2)\right\|_{M} \cdot\left\|\frac{\partial P}{\partial x_{i}}(x, y / 2)\right\|_{1} \\
& \leq C y^{-2} \varphi(y) \frac{n+1}{2 c_{n}} y^{-1}=C_{9} y^{-3} \varphi(y) .
\end{aligned}
$$


On the other hand,

$$
\begin{aligned}
\left\|\frac{\partial^{2} u}{\partial y \partial x_{i}}(x, y)\right\|_{M} & =\left\|f(x) * \frac{\partial^{2} P}{\partial y \partial x_{i}}(x, y)\right\|_{M} \\
& \leq\|f(x)\|_{M}\left\|\frac{\partial^{2} P}{\partial y \partial x_{i}}(x, y)\right\|_{1},
\end{aligned}
$$

and the equality

$$
\frac{\partial^{2} P}{\partial y \partial x_{i}}(x, y)=\frac{(n+1)(n+2)}{c_{n}} \cdot \frac{x_{i}\left(y^{2}-|x|^{2}\right)}{\left(|x|^{2}+y^{2}\right)^{(n+5) / 2}}
$$

gives

$$
\left|\frac{\partial^{2} P}{\partial y \partial x_{i}}(x, y)\right| \leq(n+1)(n+2)\left|x_{i}\right| y^{-2} P(x, y)
$$

and so

$$
\begin{aligned}
\left\|\frac{\partial^{2} u}{\partial y \partial x_{i}}(x, y)\right\|_{M} & \leq(n+1)(n+2)\left|x_{i}\right| y^{-2}\|P(x, y)\|_{1}\|f(x)\|_{M} \\
& =C_{10} y^{-2}\|f(x)\|_{M}
\end{aligned}
$$

which, in its turn, implies that

$$
\frac{\partial^{2} u}{\partial y \partial x_{i}}(x, y) \rightarrow 0 \text { as } y \rightarrow \infty
$$

Therefore

$$
\frac{\partial^{2} u}{\partial y \partial x_{i}}(x, y)=-\int_{y}^{\infty} \frac{\partial^{3} u}{\partial y^{2} \partial x_{i}}(x, s) d s
$$

which, by the assumption and equivalence (2.2), gives

$$
\begin{aligned}
\left\|\frac{\partial^{2} u}{\partial y \partial x_{i}}(x, y)\right\|_{M} & \leq \int_{y}^{\infty}\left\|\frac{\partial^{3} u}{\partial y^{2} \partial x_{i}}(x, s)\right\|_{M} d s \\
& \leq C_{9} \int_{y}^{\infty} s^{-3} \varphi(s) d s \leq C_{9} B y^{-2} \varphi(y)
\end{aligned}
$$

for all $y>0$ and $i=1,2, \ldots, n$.

(b) $\Rightarrow$ (c). The proof is similar to the proof of $(a) \Rightarrow$ (b) but here the equality

$$
\frac{\partial^{3} u}{\partial x_{j} \partial y \partial x_{i}}\left(x, y+y_{1}\right)=\frac{\partial^{2} u}{\partial y \partial x_{i}}(x, y) * \frac{\partial P}{\partial x_{j}}\left(x, y_{1}\right)
$$

is essential.

(c) $\Rightarrow(a)$. The proof is the same as that of Lemma 2.2 . 
P r o of of Theorem 3.1 (Sufficiency). Assume that $f \in L_{M}$ and the condition (3.1) holds. Let $h \in \mathbb{R}^{n}$ and $0<y<|h|$. Integrating by parts we find that

$$
\begin{aligned}
& \int_{y}^{|h|} s\left[\frac{\partial^{2} u}{\partial y^{2}}(x+h, s)+\frac{\partial^{2} u}{\partial y^{2}}(x-h, s)-2 \frac{\partial^{2} u}{\partial y^{2}}(x, s)\right] d s \\
= & |h|\left[\frac{\partial u}{\partial y}(x+h,|h|)+\frac{\partial u}{\partial y}(x-h,|h|)-2 \frac{\partial u}{\partial y}(x,|h|)\right] \\
& -y\left[\frac{\partial u}{\partial y}(x+h, y)+\frac{\partial u}{\partial y}(x-h, y)-2 \frac{\partial u}{\partial y}(x, y)\right] \\
& -\int_{y}^{|h|}\left[\frac{\partial u}{\partial y}(x+h, y)+\frac{\partial u}{\partial y}(x-h, y)-2 \frac{\partial u}{\partial y}(x, y)\right] d s \\
= & |h|\left[\frac{\partial u}{\partial y}(x+h,|h|)+\frac{\partial u}{\partial y}(x-h,|h|)-2 \frac{\partial u}{\partial y}(x,|h|)\right] \\
& -y\left[\frac{\partial u}{\partial y}(x+h, y)+\frac{\partial u}{\partial y}(x-h, y)-2 \frac{\partial u}{\partial y}(x, y)\right] \\
& -[u(x+h,|h|)+u(x-h,|h|)-2 u(x,|h|)] \\
& +u(x+h, y)+u(x-h, y)-2 u(x, y)
\end{aligned}
$$

and so,

$$
\begin{aligned}
& u(x+h, y)+u(x-h, y)-2 u(x, y) \\
= & u(x+h,|h|)+u(x-h,|h|)-2 u(x,|h|) \\
& +y\left[\frac{\partial u}{\partial y}(x+h, y)+\frac{\partial u}{\partial y}(x-h, y)-2 \frac{\partial u}{\partial y}(x, y)\right] \\
& -|h|\left[\frac{\partial u}{\partial y}(x+h,|h|)+\frac{\partial u}{\partial y}(x-h,|h|)-2 \frac{\partial u}{\partial y}(x,|h|)\right] \\
& +\int_{y}^{|h|} s\left[\frac{\partial^{2} u}{\partial y^{2}}(x+h, s)+\frac{\partial^{2} u}{\partial y^{2}}(x-h, s)-2 \frac{\partial^{2} u}{\partial y^{2}}(x, s)\right] d s \\
= & I_{1}+I_{2}-I_{3}+I_{4}
\end{aligned}
$$

Thus, by the generalized Minkowski inequality and the assumption $\alpha_{\varphi}>0$ in the 
equivalent form (2.1),

$$
\begin{aligned}
\left\|I_{4}\right\|_{M} & \leq \int_{y}^{|h|} s\left\|\frac{\partial^{2} u}{\partial y^{2}}(x+h, s)+\frac{\partial^{2} u}{\partial y^{2}}(x-h, s)-2 \frac{\partial^{2} u}{\partial y^{2}}(x, s)\right\|_{M} d s \\
& \leq 4 C \int_{y}^{|h|} s^{-1} \varphi(s) d s \\
& \leq 4 C A \varphi(|h|) .
\end{aligned}
$$

Similarly, as in the sufficiency part of the proof of Theorem 1.1 (by using the generalized Minkowski inequality, Lemma 3.2 and the assumption $\alpha_{\varphi}>0$ in the equivalent form (2.1)), we find that

$$
\begin{aligned}
\left\|I_{2}\right\|_{M} & \leq y\left\|\frac{\partial u}{\partial y}(x+h, y)-\frac{\partial u}{\partial y}(x, y)\right\|_{M}+y\left\|\frac{\partial u}{\partial y}(x-h, y)-\frac{\partial u}{\partial y}(x, y)\right\|_{M} \\
& \leq 4 y \int_{y}^{|h|}\left\|\frac{\partial^{2} u}{\partial y^{2}}(x, s)\right\|\left\|_{M} d s+2 \sum_{i=1}^{n} y \int_{0}^{\left|h_{i}\right|}\right\| \frac{\partial^{2} u}{\partial y \partial x_{i}}(x,|h|) \|_{M} d z_{i} \\
& \leq C_{11} \varphi(|h|) .
\end{aligned}
$$

Similarly,

$$
\left\|I_{3}\right\|_{M} \leq C_{12} \varphi(|h|)
$$

For the estimate of $I_{1}$ we first prove that for any real function $u$ on $\mathbb{R}^{n}$ of class $C^{2}$ and any $h \in \mathbb{R}^{n}$ we have

$$
\begin{aligned}
& u(x+h)+u(x-h)-2 u(x) \\
= & \sum_{i=1}^{n} \sum_{j=1}^{n} \int_{-1}^{1}(1-|t|) \frac{\partial^{2} u}{\partial x_{i} \partial x_{j}}(x+t h) h_{i} h_{j} d t .
\end{aligned}
$$

In fact, by the chain rule $\frac{d}{d t} u(x+t h)=\sum_{i=1}^{n} \frac{\partial u}{\partial x_{i}}(x+t h) h_{i}$, we can integrate both sides from 0 to 1 , and then integrate by parts to obtain

$$
\begin{aligned}
& u(x+h)-u(x) \\
= & \sum_{i=1}^{n} \int_{0}^{1} \frac{\partial u}{\partial x_{i}}(x+t h) h_{i} d t \\
= & \sum_{i=1}^{n}\left[\left.(t-1) \frac{\partial u}{\partial x_{i}}(x+t h) h_{i}\right|_{0} ^{1}-\sum_{j=1}^{n} \int_{0}^{1}(t-1) \frac{\partial^{2} u}{\partial x_{i} \partial x_{j}}(x+t h) h_{i} h_{j} d t\right] \\
= & \sum_{i=1}^{n} \frac{\partial u}{\partial x_{i}}(x) h_{i}+\sum_{i=1}^{n} \sum_{j=1}^{n} \int_{-1}^{1}(1-t) \frac{\partial^{2} u}{\partial x_{i} \partial x_{j}}(x+t h) h_{i} h_{j} d t .
\end{aligned}
$$

Similarly,

$$
\begin{aligned}
& u(x-h)-u(x) \\
= & -\sum_{i=1}^{n} \frac{\partial u}{\partial x_{i}}(x) h_{i}+\sum_{i=1}^{n} \sum_{j=1}^{n} \int_{-1}^{1}(1-t) \frac{\partial^{2} u}{\partial x_{i} \partial x_{j}}(x-t h) h_{i} h_{j} d t .
\end{aligned}
$$


If we add the above identities we obtain (3.2).

Now using the identity (3.2) we have for our expression $\left(I_{1}\right)$

$$
\begin{aligned}
I_{1} & =u(x+h,|h|)+u(x-h,|h|)-2 u(x,|h|) \\
& =\sum_{i=1}^{n} \sum_{j=1}^{n} \int_{-1}^{1}(1-|t|) \frac{\partial^{2} u}{\partial x_{i} \partial x_{j}}(x+t h,|h|) h_{i} h_{j} d t .
\end{aligned}
$$

Thus, by the generalized Minkowski inequality and Lemma 3.2, it yields

$$
\begin{aligned}
\left\|I_{1}\right\|_{M} & \leq\|u(x+h,|h|)+u(x-h,|h|)-2 u(x,|h|)\|_{M} \\
& \leq \sum_{i=1}^{n} \sum_{j=1}^{n} \int_{-1}^{1}(1-|t|)\left\|\frac{\partial^{2} u}{\partial x_{i} \partial x_{j}}(x+t h,|h|)\right\|_{M}\left|h_{i}\right|\left|h_{j}\right| d t \\
& \leq E \sum_{i=1}^{n} \sum_{j=1}^{n} \int_{-1}^{1}(1-|t|)|h|^{-2} \varphi(|h|)\left|h_{i}\right|\left|h_{j}\right| d t \\
& \leq n E \varphi(|h|) .
\end{aligned}
$$

Putting the above estimates togheter we obtain

$$
\|u(x+h, y)+u(x-h, y)-2 u(x, y)\|_{M} \leq C_{13} \varphi(|h|)
$$

for any $0<y<|h|$. Now, since $u(x, y) \rightarrow f(x)$ for almost all $x \in \mathbb{R}^{n}$ when $y \rightarrow 0^{+}$, we obtain (by Fatou Lemma) that $f \in Z y g\left(\varphi, L_{M}\right)$. This completes the proof.

Remark 3.3. Theorem 3.1 in the case of $L_{p}\left(\mathbb{R}^{n}\right)$-space $(1 \leq p \leq \infty)$ and with $\varphi(t)=t^{\alpha}$, where $0<\alpha<2$, was proved by STEIN ([19], Prop. 8, 8') and TAIBleson ([20], Th. 4).

Remark 3.4. For $\varphi \in P$ we have always the imbedding

$$
\operatorname{Lip}\left(\varphi, L_{M}\right) \subset Z y g\left(\varphi, L_{M}\right)
$$

but Corollary 2.3 states that if $0<\alpha_{\varphi} \leq \beta_{\varphi}<1$, then $\operatorname{Lip}\left(\varphi, L_{M}\right)=\operatorname{Zyg}\left(\varphi, L_{M}\right)$. Already ZYGMUND [23] (cf. [19], p. 148-149) observed that the space $\operatorname{Lip}\left(1, L_{\infty}\right.$ ) is strictly smaller than the space $Z y g\left(1, L_{\infty}\right)$. More examples of functions giving the strict inclusions

$$
\operatorname{Lip}\left(1, L_{p}\right) \subset Z y g\left(1, L_{p}\right) \text { and } Z y g\left(2, L_{p}\right) \subset Z y g\left(1, L_{p}\right)
$$

can be found in [19], p. 161 and [20], pp. 470-474.

Remark 3.5. In the definition of the space $\operatorname{Lip}\left(\varphi, L_{M}\right)$ we have the inequality

$$
\|f(x+h)-f(x)\|_{M} \leq C \varphi(|h|) \text { for all }|h|>0 .
$$

It is enough to have such an inequality only for small $|h|$, i.e.,

$$
\begin{aligned}
\operatorname{Lip}\left(\varphi, L_{M}\right)= & \left\{f \in L_{M}\left(\mathbb{R}^{n}\right)\right. \text { such that } \\
& \left.\|f(x+h)-f(x)\|_{M}=O(\varphi(|h|)) \text { as }|h| \rightarrow 0\right\} .
\end{aligned}
$$


Similarly

$$
\begin{aligned}
Z y g\left(\varphi, L_{M}\right)= & \left\{f \in L_{M}\left(\mathbb{R}^{n}\right)\right. \text { such that } \\
& \left.\|f(x+h)+f(x-h)-2 f(x)\|_{M}=O(\varphi(|h|)) \text { as }|h| \rightarrow 0\right\} .
\end{aligned}
$$

This observation suggests the possibility of considering the closed subspaces (analogues to the spaces $\operatorname{lip}\left(1, L_{p}\right)$ and $z y g\left(1, L_{p}\right)$ considered by ZYGMUND [24]):

$$
\begin{aligned}
\operatorname{lip}\left(\varphi, L_{M}\right)= & \left\{f \in L_{M}\left(\mathbb{R}^{n}\right)\right. \text { such that } \\
& \left.\|f(x+h)-f(x)\|_{M}=o(\varphi(|h|)) \text { as }|h| \rightarrow 0\right\} .
\end{aligned}
$$

and

$$
\begin{aligned}
z y g\left(\varphi, L_{M}\right)= & \left\{f \in L_{M}\left(\mathbb{R}^{n}\right)\right. \text { such that } \\
& \left.\|f(x+h)+f(x-h)-2 f(x)\|_{M}=o(\varphi(|h|)) \text { as }|h| \rightarrow 0\right\}
\end{aligned}
$$

\section{Some generalizations and additional remarks}

In the proof of Lemma 2.2 we used the following result, in the case when $X$ is the Orlicz space $L_{M}\left(\mathbb{R}^{n}\right)$ :

Lemma 4.1. Let $X=X\left(\mathbb{R}^{n}\right)$ be a Banach function space with the Fatou property. Then the convolution operator $(f * g)=\int_{\mathbb{R}^{n}} f(x-z) g(z) d z$ is a bounded operator from $X\left(\mathbb{R}^{n}\right) \times L_{1}\left(\mathbb{R}^{n}\right)$ into $X=X\left(\mathbb{R}^{n}\right)$ and

$$
\|f * g\|_{X} \leq\|f\|_{X}\|g\|_{1}
$$

Proof. For any $h \in X^{\prime}$ with $\|h\|_{X^{\prime}} \leq 1$ we have, by the Fubini and Hölder inequalities, that

$$
\begin{aligned}
\int_{\mathbb{R}^{n}}|(f * g)(x) h(x)| d x & \leq \int_{\mathbb{R}^{n}}\left[\int_{\mathbb{R}^{n}}|f(x-z) g(z) h(x)| d z\right] d x \\
& =\int_{\mathbb{R}^{n}}\left[\int_{\mathbb{R}^{n}}|f(x-z) h(x)| d x\right]|g(z)| d x \\
& \leq \int_{\mathbb{R}^{n}}\|f\|_{X}\|h\|_{X^{\prime}}|g(z)| d z \\
& \leq\|f\|_{X}\|g\|_{1},
\end{aligned}
$$

and, by the Fatou property of the norm,

$$
\begin{aligned}
& \|f * g\|_{X}=\|f * g\|_{X^{\prime \prime}} \\
= & \sup \left\{\int_{\mathbb{R}^{n}}|(f * g)(x) h(x)| d x \text { such that } h \in X^{\prime},\|h\|_{X^{\prime}} \leq 1\right\} \leq\|f\|_{X}\|g\|_{1} .
\end{aligned}
$$


Note that O'NEIL [15] proved the lemma above for the Orlicz spaces $L_{M}\left(\mathbb{R}^{n}\right)$ instead of $X\left(\mathbb{R}^{n}\right)$ but with the constant 2 in the estimate of the norms.

Remark 4.2. Using Lemma 4.1 we can prove Theorems 2.1 and 3.1 not only for the Orlicz spaces $L_{M}=L_{M}\left(\mathbb{R}^{n}\right)$ but even for general Banach function spaces $X=X\left(\mathbb{R}^{n}\right)$ with the Fatou property.

We consider now more general spaces $\Lambda^{k}(\varphi, X, q)$ which contain the $\operatorname{Lip}(\varphi, X)$ spaces, $Z y g(\varphi, X)$-spaces, the Stein-Taibleson $\Lambda(\alpha, p, q)$-spaces and the Herz $\Lambda(\alpha, X, q)$ spaces.

Let $\varphi \in P, 1 \leq q \leq \infty$ and let $X=X\left(\mathbb{R}^{n}\right)$ be a Banach function space with the Fatou property. The spaces $\Lambda^{k}(\varphi, X, q), k=1,2$, are the spaces of all $f \in X\left(\mathbb{R}^{n}\right)$ for which

$$
I_{\varphi, X, q}^{k}(f)=\left\{\int_{\mathbb{R}^{n}}\left(\frac{\left\|\Delta_{h}^{k} f(x)\right\|_{X}}{\varphi(|h|)}\right)^{q} \frac{d h}{|h|^{n}}\right\}^{1 / q}<\infty,
$$

with $\Delta_{h}^{1} f(x)=f(x+h)-f(x), \Delta_{h}^{2} f(x)=f(x+h)+f(x-h)-2 f(x)$, and with the norm

$$
\|f\|_{\Delta^{k}(\varphi, X, q)}=\|f\|_{X}+I_{\varphi, X, q}^{k}(f) .
$$

Note that $\Lambda^{1}\left(\varphi, L_{M}, \infty\right)=\operatorname{Lip}\left(\varphi, L_{M}\right)$ and $\Lambda^{2}\left(\varphi, L_{M}, \infty\right)=Z y g\left(\varphi, L_{M}\right)$.

Theorem 4.3. (a) The spaces $\Lambda^{k}(\varphi, X, q)$ are Banach spaces.

(b) $\Lambda^{1}(\varphi, X, q) \subset \Lambda^{2}(\varphi, X, q)$, and if $0<\alpha_{\varphi} \leq \beta_{\varphi}<1$, then $\Lambda^{1}(\varphi, X, q)=$ $\Lambda^{2}(\varphi, X, q)$.

(c) Let $f \in X\left(\mathbb{R}^{n}\right)$ and let $u$ be its Poisson integral. If $0<\alpha_{\varphi} \leq \beta_{\varphi}<k$, then $f \in \Lambda^{k}(\varphi, X, q)$ if and only if

$$
\left\{\int_{0}^{\infty}\left[\frac{y^{k}}{\varphi(y)}\left\|\frac{\partial^{k} u(x, y)}{\partial y^{k}}\right\|_{X}\right]^{q} \frac{d y}{y}\right\}^{1 / q}<\infty, \quad k=1,2 .
$$

Proof (a) If $\left(f_{n}\right)$ is a Cauchy sequence in $\Lambda^{k}(\varphi, X, q)$, then $\left(f_{n}\right)$ is obviously a Cauchy sequence in $X$, and therefore it converges in $X$ to a function $f$. Hence

$$
\left\|\Delta_{h}^{k} f_{n}(x)\right\|_{X} \longrightarrow\left\|\Delta_{h}^{k} f(x)\right\|_{X}
$$

as $n \rightarrow \infty$, and therefore, by Fatou's Lemma,

$$
I_{\varphi, X, q}^{k}(f) \leq \liminf _{n \rightarrow \infty} I_{\varphi, X, q}^{k}\left(f_{n}\right) \leq \liminf _{n \rightarrow \infty}\left\|f_{n}\right\|_{\Lambda_{\varphi, X, q}^{k}}<\infty,
$$

so that $f \epsilon_{\Lambda_{\varphi, \boldsymbol{x}, \boldsymbol{q}}^{k}}$. Further, for $m=1,2, \ldots$,

$$
\left\|\Delta_{h}^{k} f_{n}(x)-\Delta_{h}^{k} f_{m}(x)\right\|_{X} \rightarrow\left\|\Delta_{h}^{k} f(x)-\Delta_{h}^{k} f_{m}(x)\right\|_{X}
$$

as $n \rightarrow \infty$, whence, again by Fatou's Lemma,

$$
\begin{aligned}
\left\|f-f_{m}\right\|_{\Lambda^{k}(\varphi, X, q)} & \leq \liminf _{n \rightarrow \infty}\left[\left\|f_{n}-f_{m}\right\|_{X}+I_{\varphi, X, q}^{k}\left(f_{n}-f_{m}\right)\right] \\
& =\liminf _{n \rightarrow \infty}\left\|f_{n}-f_{m}\right\|_{\Lambda^{k}(\varphi, X, q)} .
\end{aligned}
$$


Since the expression on the right-hand side is arbitrarily small for all sufficiently large $m$, it follows that $f_{m} \rightarrow f$ in $\Lambda^{k}(\varphi, X, q)$, so $\Lambda^{k}(\varphi, X, q)$ is complete.

(b) The proof is the same as the proof of Corollary 2.3. We can also prove the statement by using the following equality

$$
\Delta_{h} f(x)=2^{-n} \Delta_{2^{n} h} f(x)-\sum_{k=0}^{n-1} 2^{-k-1} \Delta^{2}{ }_{2^{k} h} f\left(x+2^{k} h\right) .
$$

(c) Similarly, as in the proof of Th. 2.1 and Th. 3.2, for $f \in \Lambda^{k}(\varphi, X, q)$ and for $k=1,2$, we have that

$$
\left\|\frac{\partial^{k} u(x, y)}{\partial y^{k}}\right\|_{X} \leq C\left[y^{-n-k} \int_{0}^{y} \Omega(r) r^{n-1} d r+\int_{y}^{\infty} \Omega(r) r^{-k-1} d r\right],
$$

where

$$
\Omega(r)=\int_{S^{n-1}} \omega_{X}(r \xi) d \sigma(\xi)=\int_{S^{n-1}}\|f(x-r \xi)-f(x)\|_{X} d \sigma(\xi) .
$$

By the Hardy inequalities proved in [11] it yields that

$$
\left\{\int_{0}^{\infty}\left[\frac{y^{k}}{\varphi(y)}\left\|\frac{\partial^{k} u(x, y)}{\partial y^{k}}\right\|_{X}\right]^{q} \frac{d y}{y}\right\}^{1 / q} \leq C^{\prime}\left\{\int_{0}^{\infty}\left[\frac{\Omega(r)}{\varphi(r)}\right]^{q} \frac{d r}{r}\right\}^{1 / q}
$$

and, by the Hölder inquality,

$$
\Omega(r) \leq \sigma\left(S^{n-1}\right)^{1 / q^{\prime}}\left\{\int_{S^{n-1}} \omega_{X}(r \xi)^{q} d \sigma(\xi)\right\}^{1 / q}
$$

so that we obtain

$$
\begin{aligned}
& \left\{\int_{0}^{\infty}\left[\frac{y^{k}}{\varphi(y)}\left\|\frac{\partial^{k} u(x, y)}{\partial y^{k}}\right\|_{X}\right]^{q} \frac{d y}{y}\right\}^{1 / q} \\
\leq & C^{\prime \prime}\left\{\int_{S^{n-1}} \int_{0}^{\infty}\left[\frac{\omega_{X}(r \xi)}{\varphi(r)}\right]^{q} \frac{d r}{r} d \sigma(\xi)\right\}^{1 / q} \\
= & C^{\prime \prime}\left\{\int_{\mathbb{R}^{n}}\left[\frac{\|f(x-z)-f(x)\|_{X}}{\varphi(|z|)}\right]^{q} \frac{d z}{|z|^{n}}\right\}^{1 / q} .
\end{aligned}
$$

In the same way as in Theorems 2.1 and 3.1, we can prove the reverse inequalities by first proving the results similar to Lemmas 2.2 and 3.2 .

Remark 4.4. In STEIN [19] there are misprints in Proposition 7' and Lemma 4': conditions (61) and (62) should have $y^{1-\alpha}$ instead of $y^{\alpha-1}$. 
Remark 4.5. Considering the modulus of continuity $\omega_{1}(t, f)_{X}$ and the modulus of smoothness $\omega_{2}(t, f)_{X}$ of the function $f \in X\left(\mathbb{R}^{n}\right)$, that is,

$$
\omega_{k}(t, f)_{X}=\sup _{0<|h| \leq t}\left\|\Delta_{h}^{k} f(x)\right\|_{X^{\prime}}, \quad k=1,2,
$$

we can easily prove (cf. TAIBLESON [20]) that

$$
\Lambda^{k}(\varphi, X, q)=\left\{f \in X\left(\mathbb{R}^{n}\right):\left\{\int_{0}^{\infty}\left(\omega_{k}(t, f)_{X} / \varphi(t)\right)^{q} d t / t\right\}^{1 / q}<\infty\right\}
$$

These are the generalized Besov-Nikolskii spaces (cf. [14]). The more general spaces $\Lambda^{k}(B, X)$ were investigated by Calderón [4] and BrudnYi-Shalashov [2].

We conclude this paper remarking that results about the convolution operator and the pointwise multiplication for Lipschitz-Orlicz $\Lambda(\varphi, M, q)$ - spaces (which will contain the theorems proved in [6] and [20]) is possilble to prove by using our Theorem 4.3 (c) and the appropriate results proved by O'NEIL [15] (see also [13] and [17]) for the convolution operator and the pointwise multiplication in Orlicz spaces.

\section{References}

[1] Bergh, J. and Löfström, J: Interpolation Spaces, Springer-Verlag, Berlin-Heidelberg-New York, 1976

[2] Brudny1. Y. A. and Shalashov. V. K.: Lipschitz Spaces of Functions, Dokl. Akad. Nauk SSSR 197 (1971), 18 - 20; English transl. in Soviet Math. Dokl. 12 (1971), 383 - 386

[3] Butzer., P. L. and Berens, H.: Semi-Groups of Operators and Approximation, Springer-Verlag, New York, 1967

[4] Calderòn, A. P.: Intermediate Spaces and Interpolation, the Complex Method, Studia Math. 24 (1964), 113 - 190

[5] Flett, T. M.: Temperatures, Bessel Potentials and Lipschitz Spaces, Proc. London Math. Soc. 22 (1971), $385-451$

[6] Herz. C.: Lipschitz Spaces and Bernstein's Theorem on Absolutely Convergent Fourier Transform, J. Math. Mech. 18 (1968), 283 - 323

[7] Janson, S.: Generalization of Lipschitz Spaces and Applications to Hardy Spaces and Bounded Mean Oscillation, Duke Math. J. 47 (1980), 959 - 982

[8] Jones JR., B. F.: Lipschitz Spaces and the Heat Equation, J.Math. Mech. 18 (1968), 379 - 409

[9] Kranosel'skil, M. A. and Rutickir. Y. B.: Convex Functions and Orlicz Spaces, P. Noordhoff, Groningen 1961

[10] Krein, S. G., Petunin, Yu. I.: and Semenov, E. M.: Interpolation of Linear Operators, Providence 1982

[11] Maligr.anda, L.: Generalized Hardy Inequalities in Rearrangement Invariant Spaces, J. Math. Pures Appl. 59 (1980), 405 - 415

[12] Maligranda. L.: Indices and Interpolation, Dissertationes Math. 234, Warszawa 1985

[13] Maligranda, L.: Orlicz Spaces and Interpolation, Seminars in Math. 5, Campinas, 1989

[14] Nıкоцsкil, S. M.: Approximation of Functions of Several Variables and Imbedding Theorems, Nauka, Moscow 1977

[15] O'NeIL. R.: Fractional Integration in Orlicz Spaces, Trans. Amer. Math. Soc. 115 (1965), 300 $-328$ 
[16] Peetre, J.: New Thoughts on Besov Spaces, Duke Univ. Press 1976

[17] RaO, M. M. and ReN, Z. D.: Theory of Orlicz Spaces, Marcel Dekker, 1991

[18] Stein, E. M.: On the Functions of Littlewood-Paley, Lusin, and Marcinkiewicz, Trans. Amer. Math. Soc. 88 (1958), $430-466$

[19] Stein, E. M.: Singular Integrals and Differentiability Properties of Functions, Princeton Univ. Press, Princeton, New Jersey 1970

[20] Taibleson, M.H.: On the Theory of Lipschitz Spaces of Distributions on Euclidean $n$-Spaces. I., J. Math. Mech. 13 (1964), 407 - 479; II. ibid 14 (1965), 821 - 840; III. ibid 15 (1966), 973 981

[21] Triebel. H.: Spaces of Distributions of Besov Type on Euclidean $n$-Space. Duality, Interpolation, Arkiv Mat. 11 (1973), 13 - 64

[22] Triebel, H.: Interpolation Theory. Function Spaces. Differential Operators, VEB Deutscher Verlag der Wissenschaften, Berlin 1978

[23] Zygmund, A.: Smooth Functions, Duke Math. J. 12 (1945), 47 - 76

[24] Zygmund, A.: Trigonometric Series, Cambridge Univ. Press 1959

Department of Mathematics Claremont McKenna College Claremont, $C A 91711$

$U S A$

e-mail: aaksoy@cmcvax.claremont.edu
Department of Mathematics

Lulea University

S-971 87 Luleä

Sweden

e-mail: lech@sm.luth.se 